Dor dental nos últimos 3 meses em adolescentes

e Estratégia Saúde da Família: a comparação entre duas áreas com abordagens diferentes de atenção em saúde bucal

\author{
Dental pain in the previous 3 months in adolescents \\ and Family Health Strategy: the comparison between two areas \\ with different to oralhealth care approaches
}

Jefferson Calixto Carvalho ${ }^{1}$

M aria Augusta Bessa Rebelo ${ }^{2}$

Mario Vianna Vettore ${ }^{3}$

${ }^{1}$ Escola Superior de Ciências da Saúde, Universidade do Estado do Amazonas (ESA/UEA). Av.

Carvalho Leal 1777 ,

Cachoeirinha. 69065-001

M anaus AM .

jeff1011@hotmail.com

${ }^{2}$ Faculdade de O dontologia,

UniversidadeFederal do

Amazonas (UFAM)

${ }^{3}$ Instituto deEstudos em

Saúde Coletiva,

UniversidadeFederal do

Rio deJaneiro (IESC/UFRJ)
Abstract This study sought to compare the prevalence and intensity of dental pain in the last 3 months in adolescents living in two areas within the scope of Family $\mathrm{H}$ ealth Strategy with different to oralhealth care approaches. A cross-sectional study was conducted with 300 adolescents sel ected from public schools in the city of $M$ anaus, Brazil. Adolescents living in an area with an Oral Health Team in which of health promotion is predominant (Group I, N =150) were compared with those living in an area in which only dental treatment is offered (Group II, N=150). Demographic and socio-economic characteristics, oral health-related behavior and use of dental services were gathered. Prevalence and intensity of dental pain in the last 3 months was assessed using a tested and validated Likert scale. The mean age of the participants was $12.9 \pm 0.8$ years, of which $46.7 \%$ were male. The prevalence of dental pain in thelast 3 months was $33.7 \%$, and did not differ between Groups I and II ( $P>0.05)$. M edium to high intensity dental pain in the last 3 months was associated with Group II (OR: 2.11 $[\mathrm{Cl}$ (95\% $=1.17-3.81])$, after adjustment for covariates. Adolescents living in the area where only dental treatment is offered were more prone to severe dental pain compared to those living in the area where health programs are predominant. Key words U nified H ealth System, Dental pain, Epidemiology
Resumo 0 estudo objetivou comparar a prevalência ea intensidade da dor dental nos últimos 3 meses em adolescentes moradores de duas áreas de abrangência da Estratégia Saúde da Família com diferentes abordagens de atenção em saúde bucal. U m estudo transversal foi realizado com 300 adolescentes de 12 a 14 anos, selecionados em escolas públicas, na cidade de M anaus, Brasil. A dolescentes de uma área com Equipe de Saúde Bucal, onde são realizadas ações de promoção de saúde (Grupo I, $N=150$ ), foram comparados com moradores de uma área onde somente é oferecido (Grupo II, $\mathrm{N}=150$ ) tratamento odontológico. Características demográficas e socioeconômicas foram coletadas, bem como a prevalência e a intensidade de dor dental nos últimos 3 meses, empregando-se uma escala do tipo Likert. A média de idade dos participantes foi de $12,9 \pm 0,8$ anos, sendo $46,7 \%$ meninos. A prevalência dedor dental nosúltimos 3 meses foi de $33,7 \%$, e não diferiu entre os Grupos I ell $(P>0,05)$. A intensidadededor dental média a alta, nosúltimos 3 meses, foi associada com o Grupo II (OR: 2,11 [IC95\%=1,17-3,81]) após ajuste para covariáveis. Adolescentes moradores da área onde somente tratamento odontológico é oferecido foram mais propensos à dor dental demaior intensa em relação àqueles moradores da área onde predominam ações de promoção de saúde.

Palavras-chave Sistema Ú nico de Saúde, Dor dental, Epidemiologia 
Introdução

Dor, de forma geral, pode ser definida como "categoria de experiências múltiplaseúnicas, as quais possuem diferentes causas e características" ${ }^{\prime}$. Desse modo, a dor dental, proveniente dos dentes e estruturas adjacentes, encaixa-se nessa definição e tem como principais causas a cárie, a doença periodontal, a sensibilidade de colo e o trauma dentário, além de outros fatores de origem não-odontogênicas ${ }^{2,3}$. A dor dental também é caracterizada como um importante problema de saúde pública mundial ${ }^{4}$.

A dor dental éuma medida sensível em saúde bucal, enão deve ser considerada complementar das condições clínicas a ela associada, uma vez que nem sempre ambas estão associadas ${ }^{5}$. 0 uso demedidas dedor dental possibilita uma melhor avaliação de necessidade de cuidado e o estabelecimento de prioridades de atenção em saúde bucal. Além disso, frequentemente está associada a algum tipo de incapacidade funcional ou social. Estudos prévios demonstraram a relação entre dor dental emaior ausência na escola e no trabaIho, diminuição do convívio social, alterações do sono einterferência na capacidade mastigatória ${ }^{6}$ 12. Por conseguinte, a dor dental desencadeia custos onerosos à sociedade e afeta negativamente $a$ qualidade de vida das pessoas.

As metas globais para a saúde bucal, definidas pela Organização Mundial de Saúde para o ano de 2020, incluíram aspectos relacionados à dor dental, evidenciando sua importância para a saúde pública mundial. A redução do número de pessoas afetadas pelas limitações funcionais e pelos impactos sociais impostos pela dor de origem bucal e craniofacial, bem como a redução desses episódios são al guns dos aspectos incluídos nessas metas publicadas em $2003^{13}$.

A importância da dor dental e seus impactos na sociedade sugerem a aplicação do conhecimento sobre sua prevalência e intensidade na identificação de grupos prioritários de necessidade de cuidado nos serviços públicos de odontologia. Além disso, o emprego da dor dental como medida de saúde bucal possibilita avaliar diferentes ações e estratégias para este problema em grupos populacionais.

No Brasil, a Estratégia Saúde da Família (ESF), que se originou a partir da implantação do Sistema Ú nico deSaúdena década de 90 , constituiu a mais recente reorganização da atenção básica do serviço público no país. Essa mudança no modelo assistencial em saúde tem como proposta atividades de promoção de saúde e a apro- ximação de equipes multidisciplinares com as comunidades e famílias, consistindo na principal porta de entrada nos serviços públicos de saúde ${ }^{14,15}$. Desde 2000, a Equipe de Saúde Bucal integra a ESF, e tem como objetivo principal planejar e desenvolver atividades de promoção em saúde, além de oferecer tratamento odontológico, considerando uma visão ampliada sobre 0 processo saúde-doença. $\mathrm{N}$ esta perspectiva, 0 emprego de medidas subjetivas em odontologia, como a avaliação da dor dental, representa uma importante estratégia para que diferentes aspectos da vida dessas famílias possam ser captadas pelos profissionais em uma avaliação ampliada da saúde bucal. Essa forma de atuação permite uma maior qualidadeno planejamento das ações e na avaliação das intervenções realizadas ${ }^{16}$.

Estudos epidemiológicos analíticos em saúde bucal relativos à Equipe de Saúde Bucal na ESF são escassos na literatura, especialmente sobre dor dental. Assim, o objetivo do presente estudo foi comparar a prevalência e a intensidade desta, nos últimos 3 meses, em adolescentes moradores de duas áreas de abrangência da ESF com diferentes modelos de atenção em saúde bucal: predomínio de ações de promoção de saúde bucal (Equipe de Saúde Bucal na ESF) vs ações somente com oferta de tratamento odontológico (ESF sem Equipe de Saúde Bucal).

\section{M étodos}

Um estudo seccional de base escolar foi realizado com adolescentes de 12 a 14 anos de idade, matriculados na rede escolar pública, e residentes em duas áreas de abrangência de unidades da Estratégia Saúde da Família (ESF), no município de M anaus, AM, Brasil.

Os participantes do estudo foram selecionados em escolas referentes a duas áreas de abrangência da ESF. A exposição de interesse foi o modelo de atenção com a presença de Equipe de Saúde Bucal (ESB) naESF. Um grupo foi formado por adolescentes que residiam em uma área com ESB na ESF (Grupo I), outro grupo (Grupo II) envolveu adolescentes deumaárea deESF com acesso a serviço odontológico apenas curativo (tratamento cirúrgico-restaurador), oferecido na UnidadeBásica deSaúdeda área de abrangência.

A exposição sob estudo, presença de ESB na ESF, representa um modelo de atenção à saúde bucal que se caracteriza pela combinação deações de promoção de saúde e de tratamento odontológico. Essas ações, realizadas semanal mentepela 
ESB incluíram 3 ou mais visitas domiciliares, 4 palestras de educação em saúde e 4 escovações supervisionadas. As atividades foram realizadas para cada família, a cada 3 meses, geralmente nos domicílios. As informações foram obtidas de relatórios de produção da ESB.

A área de abrangência com ESB possuía Índice de Desenvolvimento Humano Municipal (IDHM ) igual a 0,748, efoi selecionada por conveniência e por ter a ESB na ESF implantada há dois anos (Grupo I). A área somente com oferta de tratamento odontológico (Grupo II), com IDHM igual a 0,750, foi selecionada pela semeIhança socioeconômica em relação à área do Grupo I e por nunca ter tido ESB na ESF ${ }^{17}$.

O tamanho da amostra foi calculado com base em uma prevalência de dor dental nos últimos 3 meses de $22,89 \%{ }^{6}$, com capacidade de detectar diferenças de pelo menos 10\% entreos dois grupos. Assumiu-se que adolescentes do Grupo I teriam uma prevalência de $18 \%$ de dor dental nos últimos 3 meses, e que essa prevalência nos adolescentes do Grupo II seria de 28\%. N ão foi incluída a ocorrida nos últimos 3 meses devido à não existência de dados publicados para indivíduos brasileiros. Estabeleceu-se um nível de significância 5\% eum poder de 0,80, considerando uma taxa de recusa de $15 \%$. Assim, o tamanho amostral foi definido em 150 adolescentes para cada grupo.

Inicialmente foi feito um levantamento de todas as escolas públicas existentes nos bairros e adjacências das áreas onde o estudo seria realizado. A base escolar para a seleção dos sujeitos foi escolhida devido à baixa taxa de abandono (7,3\%). Na área do Grupo I, foram identificados 186 adolescentes matriculados em 7 escolase, na área do Grupo II , 203 escolares eram elegíveis em 4 escolas. Todos os responsáveis pelos 389 escoIhidos concordaram com a participação do adolescenteno estudo, e 150 indivíduos de cada grupo foram aleatoriamente selecionados por meio de sorteio da lista das escolas.

A coleta de dados foi feita por um único entrevistador odontólogo previamente treinado e calibrado para o exame clínico realizado por outro odontólogo pesquisador, com experiência em estudos epidemiológicos. Um estudo piloto foi conduzido em uma escola pública localizada em uma área distinta daquelas do estudo principal. N essa etapa, 30 adolescentes da mesma faixa do estudo principal, foram examinados em 2 momentos. O coeficienteK appa para o índiceCPOD/ceo-d foi de 0,95 (Intervalo de Confiança (IC) de $95 \%=0,91-0,98)$, e para a necessidade de tratamento dental foi de 0,95 (IC 95\% = 0,94 0,96). Junto com a solicitação de participação no estudo, foi encaminhado um questionário autopreenchível para o responsável do adolescente. N esse questionário, foram coletadas informações demográficas dos pais, incluindo idade da mãe, escolaridade da mãe e do pai edados socioeconômicosda família, incluindo recebimento de benefício social e renda familiar. Após o recebimento do termo assinado, entrevistas individuais foram realizadas com os adolescentes com um questionário estruturado e pré testado, para a obtenção de dados demográficos: idade, sexo e raça/cor da pele; hábitos relacionados à saúde bucal: frequência de alimentação e higiene bucal noturna; uso de serviços odontológicos e avaliação da prevalência e intensidade da dor dental nosúltimos 3 meses. A raça/cor da pele foi avaliada segundo a autoclassificação dos participantes. Após as entrevistas, os sujeitos foram submetidos à exame clínicos bucais.

A prevalência dedor dental foi mensurada pela pergunta "Você sentiu dor de dentes nos últimos 3 meses?". Para avaliar a intensidade da dor dental, foi empregada uma escala do tipo Likert, validada para a população brasileira ${ }^{18}$, a qual foi aplicada somente se o adolescente respondesse positivamente ao questionamento de ter sentido dor dental nosúltimos 3 meses. A escala Likert utilizada apresenta 5 possibilidades de resposta: "não dói nada", "só um pouquinho", "mais ou menos", "muito" e"demais". N este estudo a intensidade de dor dental foi dicotomizada em "nenhuma ou baixa intensidade" (escores 0 e 1) e "média ou alta intensidade" (escores 2 a 4). A comparação da intensidade de dor dental de modo categórico foi proposta anteriormente ${ }^{8}$.

O exameclínico bucal incluiu o índice de ataque à cárie para dentes permanentes (CPO-D) e seus componentes, eo índice para dentes decíduos (ceo-d), além da frequência de dentes hígidos, cariados, restaurados e perdidos de acordo com o M anual da OM S ${ }^{19}$. Utilizou-se, nos exames clínicos, um espelho bucal plano (Probem ${ }^{\circledR}$ ) e sonda preconizada pela OMS (sonda CPI) (Stainless ${ }^{\circledR}$ ). Os exames odontológicos foram realizados nas bibliotecas das escolas onde os adolescentes estudavam, e realizados com os adolescentes e oxaminador sentados sob a luz de uma lanterna portátil fixada na região frontal do examinador ( Nautika ${ }^{\circledR}{ }^{20}$.

Cerca de $10 \%$ das entrevistas e dos exames clínicos bucais ( $\mathrm{N}=28$ ) foram realizadas em duplicata pelo mesmo entrevistador ao longo do estudo para avaliar a reprodutibilidade da aferi- 
ção de dor dental e de cárie. Para a prevalência e intensidade de dor dental nos últimos 3 meses, os coeficientes Kappa foram de 0,93 (IC de $95 \%$ $=0,86-0,99)$ e 0,63 (IC de 95\%=0,46-0,79). A concordância intraexaminador para CPO-D de Kappa foi igual a 0,95 (IC 95\% 0,94-0,96).

A normalidade da distribuição das variáveis contínuas foi avaliada pelo teste KolmogorovSmirnov. A prevalência e a intensidade da dor dental foram comparadas entre os Grupos I e II pelo teste Qui-quadrado, enquanto que as medidas nos últimos 3 meses, com diferenças estatisticamente significantes entre os grupos I ell, foram submetidas a análises bivariadas com as covariáveis. 0 Teste de Mann-Whitney foi empregado para comparar variáveis contínuas, e o TesteExato deFisher eo Teste Qui-quadrado para proporções. 0 nível designificância estabelecido para as análises bivariadas foi de $5 \%(P \leq 0,05)$.

A associação entre as diferentes abordagens de atenção em saúde bucal ea dor dental foi testada por meio de medidas de razões de chances e interval os de confiança de $95 \%$. Na análise multivariada foram incluídas todas as covariáveis com valor de $P<0,10$ da análise bivariada, empregando-se o procedimento Backward stepwise da regressão logística. Todas as análises foram realizadas no programa SPSS (Statistical Package for Social Sciences) versão 13.0.

0 presente estudo foi submetido e aprovado no Comitê de Ética em Pesquisa da Universidade Federal do Amazonas.

\section{Resultados}

A média de idade dos adolescentes foi de 12,91 \pm 0,78 anos, sendo que $46,7 \%$ eram do sexo masculino e $57,4 \%$ se declararam pardos. As informações dos pais foram obtidas para $53,7 \%$ da amostra. A média de idade das mães foi 35 anos e $35,5 \%$ das famílias recebiam benefício social. A escolaridade da mãe foi maior que a do pai nas duas áreas de ESF comparadas, predominando a renda familiar inferior a 1 salário mínimo. 0 uso deserviço odontológico foi relatado por $90 \%$ dos partici pantes, sendo que $9 \%$ estavam em tratamento e 37\% foram ao dentista no último ano (Tabela 1).

As comparações para prevalência e intensidade de dor dental nos últimos 3 meses e de características clínicas bucais para cárie dentária entre o Grupo I eo Grupo II são apresentadas na Tabela 2. A prevalência de dor dental nosúltimos 3 meses foi de $33,7 \%$, e não foi estatisticamente diferente entre os grupos. A intensidade de dor dentária média ou alta nos últimos 3 meses foi maior no Grupo II $(P<0,05)$. O CPO-D médio foi de 2,4 eo ceo- $d$ de 0,1 . Os componentes "cariado" e "restaurado" do CPO-D representaram $53,3 \%$ e $41,4 \%$ do total. A maioria dos dentes encontrava-se hígido (90,3\%), seguido da condição de cariado (4,8\%) erestaurado (3,9\%), sendo que apenas $0,6 \%$ dos dentes foram considerados como perdidos. As medidas de cárie dentária não foram estatisticamente diferentes entre os grupos. Enquanto a média de dentes restaurados nos Grupos I e II foi de 3,56 e 4,15, respectivamente $(P=0,225)$, a de dentes cariados foi de 4,42 e 5,11 ( $P=0,505)$.

As medidas de razão de chances entre a ausência deESB naESF e covariáveis ea intensidade média ou alta de dor dental nos últimos 3 meses são descritas na Tabela 3. Foram incluídas na análise multivariada as variáveis Grupo II, idade do adolescente e alimentação antes de dormir. A chance de relatar intensidade média ou alta de dor dental nos últimos 3 meses foi 2,11 vezes maior entre aqueles que moravam na área sem ESB na ESF quando comparados com aqueles que moravam na área com ESB na ESF. A alimentação, às vezes, antes de dormir, permaneceu associada com a intensidade média ou alta de dor dental nos últimos 3 meses [OR: 3,42 IC 95\%: 1,44-8,10].

\section{Discussão}

A modificação do modelo de atenção em saúde bucal nos serviços públicos, a partir da inserção da Equipe de Saúde Bucal na atenção básica por meio da Estratégia deSaúdeda Família, representou uma importante conquista. Isso porque as práticas de atenção à saúde, anteriormente centradas em procedimentos restauradores e curativos, passaram a ter como foco ações de promoção da saúde e prevenção de agravos ${ }^{21}$. Os estudos avaliativos sobre a Equipe de Saúde Bucal na Estratégia de Saúde da Família têm sido concentrados em pesquisas sobre satisfação dos usuári0s ${ }^{22-24}$, perfil de implantaçãa $25-29$, tipos de ações de saúde bucal desenvolvidas na ESF ${ }^{23,28}$ e critérios para definição degrupos com maiores necessidades de atenção em saúde bucal ${ }^{30}$. A pesar da ruptura do antigo modelo de serviço odontológico tradicional, são inexistentes estudos que compararam possíveis diferenças epidemiológicas em saúde bucal entre populações com e sem Equipe deSaúde Bucal na Estratégia deSaúdedaFamília. 
Tabela 1. Características demográficas e socioeconômicas nas áreas com Equipe de Saúde Bucal na Estratégia Saúde da Família (Grupo I) e sem Equipe de Saúde Bucal na Estratégia Saúde da Família (Grupo II).

\begin{tabular}{|c|c|c|c|}
\hline Características demográficas e soci oeconômicas & $\begin{array}{l}\text { Grupo I } \\
(\mathrm{N}=150)\end{array}$ & $\begin{array}{l}\text { Grupo II } \\
(\mathrm{N}=150)\end{array}$ & Total \\
\hline \multicolumn{4}{|l|}{ Adolescentes } \\
\hline Idade, média ( \pm DP) & $13,01 \pm 0,80$ & $12,81 \pm 0,75$ & $12,91 \pm 0,78$ \\
\hline \multicolumn{4}{|l|}{ Sexo, $n(\%)$} \\
\hline M asculino & $77(51,3)$ & $63(42,0)$ & $140(46,7)$ \\
\hline Feminino & $73(48,7)$ & $87(58,0)$ & $160(53,3)$ \\
\hline \multicolumn{4}{|l|}{ Raça, n (\%) } \\
\hline Branca & $41(30,1)$ & $51(34,9)$ & $92(32,6)$ \\
\hline Parda & $86(63,2)$ & $76(52,1)$ & $162(57,4)$ \\
\hline Preta & $9(6,7)$ & $19(13,0)$ & $28(10,0)$ \\
\hline \multicolumn{4}{|l|}{ Pais* } \\
\hline I dade da mãe, média ( \pm DP) & $35,92 \pm 6,08$ & $33,81 \pm 5,30$ & $35,09 \pm 5,86$ \\
\hline \multicolumn{4}{|l|}{ A família recebe benefício social, $\mathrm{n}(\%)$} \\
\hline Sim & $28(29,5)$ & $27(45,0)$ & $55(35,5)$ \\
\hline Não & $67(70,5)$ & $33(55,0)$ & $100(64,5)$ \\
\hline \multicolumn{4}{|l|}{ Escolaridade do pai, em anos, n (\%) } \\
\hline Até 7 & $48(54,5)$ & $29(48,3)$ & $77(52,0)$ \\
\hline De 8 a 10 & $21(23,9)$ & $8(13,4)$ & $29(19,6)$ \\
\hline 11 ou mais & $19(21,6)$ & $23(38,3)$ & $42(28,4)$ \\
\hline \multicolumn{4}{|l|}{ Escolaridade da mãe, em anos, n (\%) } \\
\hline Até 7 & $26(31,7)$ & $11(19,6)$ & $37(26,8)$ \\
\hline De 8 a 10 & $20(24,4)$ & $13(23,2)$ & $33(23,9)$ \\
\hline 11 ou mais & $36(43,9)$ & $32(57,2)$ & $68(49,3)$ \\
\hline \multicolumn{4}{|l|}{ Renda familiar, n (\%) } \\
\hline$<1 \mathrm{SM}$ & $41(50,7)$ & $31(60,8)$ & $72(54,5)$ \\
\hline 1 a 3 SM & $30(37,0)$ & $16(31,4)$ & $46(34,8)$ \\
\hline$>3 \mathrm{SM}$ & $10(12,3)$ & $4(7,8)$ & $14(10,7)$ \\
\hline \multicolumn{4}{|l|}{ Uso do serviço odontológico, n (\%) } \\
\hline $\operatorname{Sim}$ & $136(90,7)$ & $134(89,3)$ & $270(90)$ \\
\hline Não & $14(9,3)$ & $16(10,7)$ & $30(10)$ \\
\hline \multicolumn{4}{|l|}{ Tempo desde a última consulta odontológica, n (\%) } \\
\hline Em tratamento & $12(8)$ & $16(10,7)$ & $28(9,3)$ \\
\hline$<6$ meses até 1 ano & $60(40)$ & $51(34)$ & $111(37)$ \\
\hline$>1$ ano & $27(18)$ & $18(12)$ & $45(15)$ \\
\hline Não lembra & $37(24,7)$ & $49(32,6)$ & $86(28,7)$ \\
\hline
\end{tabular}

SM : salário mínimo; *Para as informações obtidas dos pais o n foi de 161.

Neste estudo, a comparação da dor dental entre áreas com e sem Equipe de Saúde Bucal na Estratégia de Saúde da Família teve como objetivo avaliar se diferentes modelos de atenção estariam associados com o principal desfecho nas atividades diárias e na qualidade de vida.

0 principal achado deste estudo foi a associação entre a ausência de ESB na ESF e a intensidade de dor dental média ou alta nos últimos 3 meses em adolescentes. A pesar de não terem sido avaliadas as ações da ESB na ESF no Grupo I, esteresultado pode, ao menos em parte, ser atribuído às diferentes práticas de atenção à saúde bucal. Considerando que a utilização de serviços odontológicos e o tempo desde a última consulta foi similar entre os adolescentes dos grupos estudados, as ações de promoção de saúde complementares ao tratamento curativo, realizadas na área com ESB na ESF, podem ter sido responsáveis pela menor ocorrência de dor dental média ou alta nesse grupo. 0 desenvolvimento de ações de promoção de saúde bucal adequadas ao contexto social local, em uma abordagem populacional, pode promover mudanças ambientaise de comportamento, favorecendo-a, mas o pouco tempo de instalação da ESB na área (2 anos) contraria essa premissa. É mais provável que 0 diagnóstico precocee o pronto atendimento, por 
Tabela 2. Características clínicas bucais e dor dental e intensidade de dor dental nos últimos 3 meses em adolescentes em áreas com Equipe de Saúde Bucal na Estratégia Saúde da Família (Grupo I) e sem Equipe de Saúde Bucal na Estratégia Saúde da Família (Grupo II).

\begin{tabular}{|c|c|c|c|c|}
\hline & Grupo I & Grupo II & Total & Valor de $P$ \\
\hline Dor dental nos últimos 3 meses, n (\%)** & & & & 0,179 \\
\hline Sim & $45(30)$ & $56(37,3)$ & $101(33,7)$ & \\
\hline Não & $105(70)$ & $94(62,7)$ & $199(66,3)$ & \\
\hline \multicolumn{5}{|l|}{ Intensidade dor dental nos últimos 3 meses, n (\%)** } \\
\hline Nenhuma ou baixa & $23(51,1)$ & $16(28,6)$ & $39(38,6)$ & 0,021 \\
\hline Média ou alta & $22(48,9)$ & $40(71,4)$ & $62(61,4)$ & \\
\hline CPO-D, média $( \pm D P)^{*}$ & $2,23 \pm 2,41$ & $2,62 \pm 2,70$ & $2,42 \pm 2,55$ & 0,176 \\
\hline \multicolumn{5}{|c|}{ Frequência de componentes do CPO-D, média $( \pm D P)^{*}$} \\
\hline Cariado & $54,42 \pm 44,17$ & $52,19 \pm 41,94$ & $53,29 \pm 42,97$ & 0,770 \\
\hline Restaurado & $39,85 \pm 42,56$ & $42,95 \pm 40,43$ & $41,42 \pm 41,43$ & 0,541 \\
\hline Perdido & $5,73 \pm 17,27$ & $4,87 \pm 12,76$ & $5,29 \pm 15,12$ & 0,946 \\
\hline ceo-d, média $( \pm D P)^{*}$ & $0,07 \pm 0,36$ & $0,03 \pm 0,21$ & $0,05 \pm 0,30$ & 0,511 \\
\hline Frequência de dentes hígidos, média ( $\pm \mathrm{DP})^{*}$ & $91,27 \pm 8,62$ & $89,27 \pm 10,32$ & $90,27 \pm 9,54$ & 0,109 \\
\hline Frequência de dentes cariados, média ( $\pm \mathrm{DP}) *$ & $4,42 \pm 6,20$ & $5,11 \pm 6,93$ & $4,76 \pm 6,57$ & 0,505 \\
\hline Frequência de dentes restaurados, média ( $\pm \mathrm{DP})^{*}$ & $3,56 \pm 6,24$ & $4,15 \pm 6,03$ & $3,86 \pm 6,13$ & 0,225 \\
\hline Frequência de dentes perdidos, média, ( $\pm \mathrm{DP})^{*}$ & $0,57 \pm 2,01$ & $0,54 \pm 1,62$ & $0,56 \pm 1,82$ & 0,868 \\
\hline
\end{tabular}

Valor de P refere-seao Teste Q ui-quadrado (**) eteste M ann-Whitney $\left({ }^{*}\right)$.

Tabela 3. Análises brutas e ajustadas entre ausência de Equipe de Saúde Bucal na Estratégia de Saúde da Família e intensidade de dor dental média ou alta nos últimos 3 meses.

\begin{tabular}{lcccc}
\hline & OR bruta & IC95\% & OR ajustada & IC95\% \\
\hline Idade & 0,73 & $0,50-1,05$ & - & - \\
Característica da ESF & & & & - \\
$\quad$ Com ESB (Grupo I) & 1 & - & 1 & - \\
$\quad$ Sem ESB (Grupo II) & 2,12 & $1,19-3,78$ & 2,11 & $1,17-3,81$ \\
Alimentação antes de dormir & & & 1 & - \\
$\quad$ Não & 1 & - & 3,45 & $1,44-8,27$ \\
Sim, às vezes & 3,42 & $1,44-8,10$ & 1,23 & $0,56-2,70$ \\
Sim, sempre & 1,21 & $0,56-2,63$ & & \\
\hline
\end{tabular}

meio de restaurações em lesões menores, tenham reduzido a dor dental.

A prevalência de dor dental de $33,7 \%$ nos últimos 3 meses, encontrada neste estudo, foi semelhante à de anteriores. No último inquérito nacional de saúde bucal, a prevalência de dor dental nos últimos 6 meses, para adolescentes de 15 a 18 anos, foi de $35,6 \%{ }^{31}$, enquanto que num estudo em escolares de 14 e 15 anos, em Recife, esta foi de 33,6\% ${ }^{32}$. Em Florianópolis, a dor dental nos últimos 12 meses, em adolescentes de 12 a 13 anos, foi de $33,7 \%{ }^{33}$.

A percepção sobre a intensidade de dor dental não é consenso na literatura. N este estudo, a intensi dade de dor dental média ou alta nosúltimos 3 meses foi de $20,7 \%$. No último inquérito nacional, a intensidade de dor dental média ou alta nos últimos 6 meses foi de $46,4 \%$ entre adolescentes de 15 a 18 anos $^{31}$. Em outros estudos, os escores mais altos de intensidade de dor foram os mais relatados, independentemente do período recordatório, como em escolares de 8 anos da Inglaterra, em que a dor dental foi considerada muito intensa por $40 \%$ das crianças ${ }^{11}$, e em crianças sul-africanas, ondeo escoremais elevado foi o que obtevea maior frequência $(38 \%)^{34}$. No Brasil, um estudo com adolescentes escolares de 14 a 15 anos, em Pernambuco, verificou 
maiores percentuais para dor dental média ou alta nos últimos 6 meses $(64,1 \%)^{32}$. Em Santa Catarina, a maior porcentagem de intensidade de dor dental em escolares de 12 anos foi a de intensidade média ${ }^{6}$. Em outro estudo, as intensidades de dor dental baixa e alta foram semeIhantes em escolares de 8 a 9 anos deidadematriculados em unidades públicas e particulares ${ }^{35}$. Possíveis explicações para as diferenças na intensidade de dor dental entre o presente estudo e os demais podem ser devido ao período de recordatório da dor dental, ou, ainda, devido a dife renças no perfil socioeconômico da população, uma vez que a ocorrência de dor dental tem sido associada ao baixo nível social e econômico no Brasi| ${ }^{33,36 .}$

Aspectos metodológicos corroboram a validade interna do presente estudo. 0 emprego de uma escala para avaliação de dor validada, para a população do estudo e aplicada por um odontólogo previamente treinado e calibrado, evitou possíveis vieses de aferição do desfecho. A reaplicação da escala de dor ao longo do estudo, em $10 \%$ da amostra, revelou uma adequada reprodutibilidade do instrumento empregado. Outro aspecto metodológico relevante para comparar diferentes modelos de atenção em saúde bucal com a dor dental foi a escolha prévia de áreas com IDHM similares ${ }^{17}$. Assim, a potencial confusão de algumas variáveis socioeconômicas foi minimizada. Além disso, o emprego de análise multivariada permitiu observar a associação entreausência deESB na ESF ea intensidade de dor dental média ou alta nosúltimos 3 meses, ajustada para covariáveis. 0 delineamento transversal desteestudo éuma limitação, não possibilitando inferências causais entre o modelo de atenção exclusivamente terapêutico com maior ocorrência de intensidade média ou alta de dor dental nos últimos 3 meses. Outra limitação foi a taxa de resposta aos questionários enviados aos responsáveis dos adolescentes $(53,7 \%)$.

Apesar das atividades de promoção de saúde bucal desenvolvidas pelas ESB na ESF serem normatizadas pelo M inistério da Saúde, o estudo de duasáreas deabrangência deESF em M anaus, AM , poder refletir características específicas locais em relação à política de saúde bucal do município.

Os resultados obtidos no presente estudo permitem concluir que adolescentes moradores da área onde somente tratamento odontológico é oferecido foram mais propensos à dor dental mais intensa nos últimos 3 meses em relação àqueles moradores da área onde existe ESB na ESF, caracterizada pelo predomínio de ações de promoção de saúde. Estudos do tipo ensaio comunitário são necessários para avaliar os reais efeitos de diferentes práticas em saúde bucal sobreo perfil epidemiológico entreáreas com esem ESB na ESF.

\section{Colaboradores}

JC Carvalho, M AB Rebelo e M V Vettore participaram igualmente de todas as etapas da elaboração do artigo.

\section{Referências}

1. Melzack R, Wall PD. The challenge of pain. London: Penguin Books; 1996.

2. Antunes JLF, Peres MA, Crivello Júnior O. Epidemiologia da saúde bucal. Rio de Janeiro: Guanabara Koogan; 2006.

3. Luiz RR, Costa AJL, Nadanovsky P. Epidemiologia e bioestatística na pesquisa odontológica. São Paulo: Atheneu; 2005.

4. Góes PSA, Kosminsky M, Siqueira JTT, Ribeiro M FP. Dor orofacial. In: Antunes JLF, Peres MA, Crivello Júnior 0 , organizadores. Epidemiologia da saúde bucal. Rio de Janeiro: Guanabara Koogan; 2006. p. 102-114.

5. Nadanovsky P, Costa AJL. Indicadores de saúde bucal. In: Luiz RR, Costa AJL, Nadanovsky P, organizadores. Epidemiologia e bioestatística na pesquisa odontológica. São Paulo: Atheneu; 2005. p. 143-164

6. Hack-Comunello SM, Michel-Crosato E, Biazevic M GH, Crosato E. Dor dental e condição sócioeconômica: um estudo censitário em escolares. Pesa Bras Odontoped Clin Integr 2008; 8(1):63-67. 
7. Guerunpong S, Tsakos G, Sheiham A. The prevalence and severity of oral impacts on daily performances in Thai primary school children. Health Qual Life Outcomes 2004; 2(57):1-8.

8. M acfarlane TV, Blinkhorn AS, Davies RM, Kincey $\mathrm{J}$, Worthington HV. Oro-facial pain in the community: prevalence and associated impact. Community Dent Oral Epidemiol 2002; 30(1):52-60.

9. Slade GD. Epidemiology of dental pain and dental caries among children and adolescents. Community Dent $\mathrm{H}$ ealth 2001; 18(4):219-227.

10. Bassols A, Bosch F, Campillo M, Cañellas M, Baños JE. An epidemiological comparison of pain complaints in the general population of Catalonia (Spain). Pain 1999; 83(1):9-16.

11. Shepherd MA, Nadanovsky P, Sheiham A. The prevalence and impact of dental pain in 8-year-old school children in Harrow, England. Br Dent J 1999; 187(1):38-41.

12. Reisine ST. The effects of pain and oral health on the quality of life. Community Dent $\mathrm{H}$ ealth 1988; $5(1): 63-68$.

13. Hobdell $M$, Petersen PE, Clarkson J, Johnson N. Global goals for oral health 2020. Int Dent J 2003; 53(5):285-288.

14. Rosa WAG, Labate RC. Programa saúde da família: a construção de um novo modelo de assistência. Rev Latino-Am Enfermagem 2005; 13(6):1027-1034.

15. Escorel S, Giovanella L, M endonça MHM, Senna M CM . O Programa de Saúde da Família e a construção de um novo modelo para a atenção básica no Brasil. Rev Panam Salud Pública 2007; 21(2):164-176.

16. Aerts D, Abegg C, Cesa K. O papel do cirurgiãodentista no Sistema Único de Saúde. Cien Saude Colet 2004; 9(1):131-138.

17. Amazonas. Secretaria de Estado de Planejamento e Desenvolvimento Econômico. Atlas do Desenvolvimento Humano em M anaus; 2006.

18. Lamarca GA, M onteiro da Silva AM. Escala likert de dor para crianças: um estudo preliminar de validação. In: Anais do 5o Simpósio Brasileiro e Encontro Internacional sobre Dor; 2001; São Paulo. São Paulo: Lemos Editorial; 2001. p. 282.

19. World Health Organization (WHO). Oral Health Surveys: basic methods. 4 ed. Geneva: ORH/EPI; 1997.

20. Brasil. Ministério da Saúde. Secretaria de Políticas de Saúde. Projeto SB2000: condições de saúde bucal da população brasileira no ano 2000: manual do examinador. Brasília, DF: M inistério da Saúde; 2001.

21. Brasil. M inistério da Saúde/SAS. Diretrizes da política nacional de saúde bucal. Brasília, DF: Ministério da Saúde; 2004.

22. Andrade KLC, Ferreira EF. Avaliação da inserção da odontologia no Programa Saúde da Família de Pompéu (M G): a satisfação do usuário. Cien Saude Colet 2006; 11(1):123-130.

23. Emmi DT, Barroso RFF. Avaliação das ações de saúde bucal no Programa Saúde da Família no distrito de Mosqueiro, Pará. Cien Saude Colet 2008; 13(1):35-41.
24. Oliveira AKP, Borges DF. Programa de Saúde da Família: uma avaliação de efetividade com base na percepção de usuários. Rev. Administração Pública 2008; 42(2):369-389.

25. Baldani MH, Fadel CB, Possamai T, Queiroz MGS. A inclusão da odontologia no Programa Saúde da Família no Estado do Paraná, Brasil. Cad Saude Publica 2005; 21(4):1026-1035.

26. Canesqui AM, Spinelli MAS. A implementação do Programa Saúde da Família em municípios do Estado de M ato Grosso, Brasil. Cad Saude Publica 2008; 24(4):862-870.

27. Henrique F, Calvo MCM. Avaliação do Programa Saúde da Família nos municípios do Estado de Santa Catarina, Brasil. Cad Saude Publica 2008; 24(4):809819.

28. Oliveira JLC, Saliba NA. Atenção odontológica no Programa de Saúde da Família de Campos dos Goytacazes. Cien Saude Colet 2005; 10(Supl.):297-302.

29. Souza TM, Roncalli AG. Saúde bucal no Programa Saúde da Família: uma avaliação do modelo assistencial. Cad Saude Publica 2007; 23(11):2727-2739.

30. Terreri ALM, Soler ZASG. Estudo comparativo de dois critérios utilizados no Programa Saúde da Família na priorização do tratamento da cárie entre crianças de 5 a 12 anos. Cad Saude Publica 2008; 24(7):1581-1587.

31. Brasil. M inistério da Saúde/SAS. Projeto SB-Brasil 2003: condições de saúde bucal da população brasileira 2002-2003. Brasília, DF: M inistério da Saúde; 2004.

32. Goes PS, Watt R, Hardy RG, Sheiham A. The prevalence and severity of dental pain in 14-15 year old Brazilian schoolchildren. Community Dent $\mathrm{H}$ ealth 2007; 24(4):217-224.

33. Nomura LH, Bastos JLD, Peres MA. Dental pain prevalence and association with dental caries and socioeconomic status in schoolchildren, Southern Brazil, 2002. Braz Oral Res 2004; 18(2):134-140.

34. Naidoo S, Chikte UM, Sheiham A. Prevalence and impact of dental pain in 8-10-year-olds in the Western Cape. SADJ 2001; 56(11):521-523.

35. Barreto EP, Ferreira E, Ferreira E, Pordeus IA. Evaluation of toothache severity in children using a visual analogue scale of faces. Pediatric dentistry 2004; 26(6):485-491.

36. Bastos JLD, Nomura LH, Peres MA. Dental pain, socioeconomic status, and dental caries in young male adults from southern Brazil. Cad Saude Publica 2005; 21(5):1416-1423.

Artigo apresentado em 15/07/2010

Aprovado em 08/09/2010

Versão final apresentada em 01/10/2010 